\title{
THE MODIFICATIONS PRODUCED IN THE
} TEMPERATUURE OF THE BODY

BY THE

\section{LOCAL APPLICATION OF COLD AND HEAT.}

BY

FREDERICK BARHAM NUNNELEY, M.D., LECTORER ON EXPERIMENTAL PHYSIOLOGY AT ST. MARY'S HOSPITAL; ASSISTANT-PHYSICIAN TO ST. MARY's HOSPITAL AND TO THE HOSPITAL FOR IICK CHILDREN.

Received May 30th.-Read June 27th, 1871.

THE inflammation of internal organs is ascribed with great frequency, both popularly and by the physician, to the effect of cold acting externally, and especially to its local influence in the form of draughts and chills, such as are caused by currents of cold air, wet feet, \&c., when the body is over-heated or is felt to be so. At the same time it is well known that the heat of the body remains nearly constant, notwithstanding great variations of the surrounding temperature.

With these facts in view, it becomes a matter of some interest to determine whether, under any circumstances, local applications of cold modify the temperature of the body generally, or of any part of it other than that on which they immediately act; or, in other words, whether any disturbance of the conditions regulating the natural heat of the body can 
be induced in a moderate time by these means. The observations related below deal with some of these circumstances, and though a part of the ground has been gone over before by Edwards ${ }^{1}$ and by Tholozan and Brown-Séquard, ${ }^{2}$ my results differ so much from theirs that it seemed better to examine the matter afresh. In doing this three questions presented themselves for solution, to which direct experiment only could supply an answer, viz. what effect is produced on the temperature of any part of the body when one of the extremities is placed-1, in cold water; or, 2 , in hot water ; or, 3, when immersion in hot is immediately followed by immersion in cold water?

The extremities only are referred to, since they are well adapted for observations of this kind, and their temperature can be determined with great accuracy. From its convenience, water was used as the source of heat and cold.

The observations were thus conducted.

The subject of experiment was placed in a room free from draughts and of such temperature that no feelings of cold or heat were experienced, since these, as it will appear later on, seem to have a marked influence on the result. For similar reasons the cold and hot water were brought to him. A thermometer easily readable to $0 \cdot 1 \mathrm{~F}$. was placed in one or other hand (or foot) and the fist supported for a time by an elastic band stretched around it. When the mercury became stationary, which usually happened in 20-30 minutes, the other hand or foot was put into cold or hot water and maintained there for 15-40 minutes, and the temperature of the unimmersed hand noted at short intervals.

Brown-Séquard ${ }^{3}$ directs that observations of two minutes each should be taken of the unimmersed hand, the one before and the other after immersion of the opposite hand, in order that as far as possible the temperature of the exposed palm

1 “Animal Heat," in 'Todd's Cyclopædia of Anatomy and Physiology,' vol. ii, p. 660, 1839.

2 'Journal de la Physiologie,' t. i, p. 500 et seq., 1858. 'Experimental Researches applied to Physiology and Pathology,' p. 32, 1853.

3 'Journal de la Physiologie,' t. i, p. 504. 
may be ascertained; but this method seemed objectionable, inasmuch as it rendered it impossible to determine if a rise of temperature took place from an application to the opposite hand, as such would necessarily be the course of the mercury for several minutes more.

The heat of the body generally was examined by means of a thermometer placed under the tongue.

The experiments are arranged in a tabular form at the end of the paper, and reference will be made to them by numbers.

To proceed with the questions raised.

1. It was found in different individuals, male and female, that when one hand or foot was put into water ranging in temperature from $50^{\circ}$ to $25^{\circ} \mathrm{F}$., and maintained there for 4-40 minutes, no change occurred in the temperature of the body generally or of the other extremities. And further, that if the temperature of the unimmersed hand had not attained its maximum, it still continued to rise notwith. standing the cold applied to the other. (Exps. I, II, III and VI-XI.)

In one individual the sensation of pain was so acute as almost to mask that of cold, and yet the temperature did not fall in the opposite hand. (Exp. IV.) Similar results were obtained with others to whom cold water gave comparatively little pain, and markedly so in a lady who kept one hand immersed uninterruptedly for half an hour in water containing melting ice, the temperature of the other hand steadily rising for twenty-three minutes of this time. (Exp. V.)

A curious variation of this result was obtained in the same case on another occasion, when the subject of it was tired and the room sensibly colder than before. (Exp. VII.)

This last observation well illustrates some important points. 1. That nervous exhaustion of the subject of experiment, and coldness of the surrounding air, cause a fall in temperature which does not occur without them. 2. That this fall in temperature is not often coincident with a general sensation of coldness. 3. That no fall of temperature, so prompt as to voL. LIV. 
be fairly considered as a reflex act, follows the immersion of one hand in a freezing mixture.

2. When one hand was put into water as hot as it could be borne, which was with most persons from $110-120^{\circ} \mathrm{F}$., it was found that the temperature of the other hand and of the feet rose from $0.8^{\circ}$ to $3.4^{\circ}$, and that of the mouth under the tongue from $0.3^{\circ}$ to $0.9^{\circ}$, the rise in the opposite hand and in the foot being equal. (Exps. XII, and XIV-XXIII.) The increase of heat was accompanied by a general sensation of warmth, sweat often breaking out on the unimmersed hand, and even over the body. These effects varied considerably in degree with the individual, and even in the same individual at different times. The temperature of the mouth rose in the case of the lady referred to above from $98.4^{\circ}$ to $99.4^{\circ}$. (Exp. XIII.)

3. When hot water had produced the maximum effect just referred to, if the hand was removed, and it or one of the other extremities plunged into cold water and maintained there for some time, it was found that the temperature of the unimmersed hand and of the feet began to fall, and would in twenty or thirty minutes sink from $1^{\circ}$ to $7 \cdot 5^{\circ}$, the fall commencing very shortly after immersion. At the same time the temperature of the mouth would decrease from $0 \cdot 6^{\circ}$ to $1 \cdot 4^{\circ}$. (Exps. XIII to XXIII.) A trifling sensation of chilliness was often coincident with this loss of heat, and persisted whilst it continued. A kind of reaction then took place, the chilliness passed off and the temperature rose; this could be hastened by warm tea, \&c., and especially by immersion of an extremity in hot water; the latter method would in a short time arrest the fall of temperature when this was still going on and would again cause a rise, as just described, somewhat beyond the natural bounds. Experiments XIV and XVI illustrate this.

The results of these experiments may be thus summed up. 
1. No change.occurs in the temperature of any part of the body from the local application of cold, except that on which it immediately acts, unless the individual is in a state of more or less nervous exhaustion or there is decided coolness of the surrounding air.

2. The local application of heat exceeding in degree that of the blood causes a slight rise of the general temperature and a more considerable one of that of the extremities.

3. If cold is now applied locally-as to any one of the extremities-the general temperature falls slightly and that of the extremities more notably below the natural standard, but both this fall and the previous rise with heat vary considerably, not, however, with the individual susceptibility to painful sensations of temperature, but rather in proportion to the facility with which the conditions regulating the normal balance of the bodily temperature can be disturbed.

4. This change, unlike a reflex act, occurs comparatively, or even very slowly, and its amount is equal in all the extremities; hence it is not, at any rate in many cases, a reflex action, taking place between homologous parts, as BrownSéquard has described it. ${ }^{1}$

Perhaps these changes of temperature may be regarded as parallel in many respects with those attending a slight rigor. In both cases a disturbance, probably of the nervous system, sufficient to derange the conditions presiding over the maintenance of the natural temperature, seems first of all necessary. This may be due to heat, exhaustion from over-work, or want of food, or, in the case of disease, to a morbid poison. When the nervous system is thus, so to speak, taken at a disadvantage, slight impressions of cold may produce a rigor, as at the onset of pneumonia, or lowering of the heat of the extremities with slight chilliness, as in the artificial conditions related above. Even a true rigor, caused by a morbid poison, may be prolonged or induced by exposure to cold. ${ }^{2}$

1 'Journal de la Physiologie,' t. i, p. 505.

2 On the subject of rigors see Wunderlich, 'Temperature in Disease,' Syd. Soc. Translation, pp. 172-199. 
In both cases reaction from a state of lowered temperature occurs; in a true rigor the heat passes the natural limit and pyrexia is established, whilst in these experiments the normal temperature was slowly attained.

It would be undesirable to do more than suggest such an explanation, especially as the experiments of Dr. BrownSéquard afforded such different results. It has been my wish rather to bring the facts observed under notice in such form as might best connect them with other and known phenomena, than to attempt to explain all the variations which occurred.

The cold air in which Brown-Séquard made his observations would, no doubt, modify the results; indeed, I have found this to be the case by actual experiment. (Exp. IX, b.)

Although the actual observations scarcely support Dr. W. F. Edwards's axiom, "1 "That we cannot either raise or lower the temperature of any one part of the body without all the other parts of the frame being affected and suffering a corresponding rise or fall in temperature, more or less, according to circumstances," as a general rule, they bear it out in particular cases and subject to certain conditions.

Dr. Handfield Jones ${ }^{2}$ states that he has found that plunging one hand into hot water causes a speedy fall in the temperature of the opposite one. In my experience this was a comparatively rare occurrence, and did not usually exceed $0.1^{\circ}$ or $0.2^{\circ} \mathrm{F}$. (Exps. XIII and XVI.) In one instance, however, immersion of a foot in hot water caused an immediate fall in the temperature of the hand to the extent of $3 \cdot 6^{\circ}$. (Exp. XVIII.) This had all the appearance of a reflex act, but,-and the remark applies to the whole subject of the paper,-this field of inquiry is so incompletely explored that we can well suppose the same end may be attained by different paths - that such changes of temperature may occur in some cases as the analogues of trifling rigors, in others by reflex agency. The practical lesson which the facts

1 'Todd's Cyclop.,' loc. cit.

2 'Functional Nervous Disorders,' 2nd ed., p. 34. 
teach us, is that partial chills can modify the temperature, and that a condition of depressed nervous energy is one in which an individual is particularly exposed to the effects of slight impressions of cold.

The temperatures in the following tables are those of Fahrenheit's thermometer.

The extremes of temperature in each experiment, and the difference between them, have been printed in bold type for convenience of reference. 


\begin{tabular}{|c|c|c|c|c|c|c|c|c|c|}
\hline \multicolumn{6}{|c|}{$\begin{array}{l}\text { OBSERVATION OF TEMPERATURE PREVIOUS } \\
\text { TO APPLICATION OF' COLD. }\end{array}$} & \multicolumn{4}{|c|}{ APPLICATION OF COLD. } \\
\hline $\begin{array}{l}\text { Experi- } \\
\text { ment. }\end{array}$ & $\begin{array}{c}\text { Part of body } \\
\text { in which } \\
\text { tempera- } \\
\text { ture was } \\
\text { observed. }\end{array}$ & $\begin{array}{l}\text { Tempe- } \\
\text { rature of } \\
\text { room. }\end{array}$ & $\left|\begin{array}{l}\text { Tempe- } \\
\text { rature at } \\
\text { the end } \\
\text { of } 5-10 \\
\text { minutes. }\end{array}\right|$ & $\begin{array}{l}\text { Tempe- } \\
\text { rature at } \\
\text { the end } \\
\text { of } 10-20 \\
\text { minutes. }\end{array}$ & $\begin{array}{c}\text { Tempe- } \\
\text { rature at } \\
\text { the end } \\
\text { of } 20-40 \\
\text { minutes. }\end{array}$ & $\begin{array}{l}\text { Name of } \\
\text { extremity } \\
\text { subjected } \\
\text { to cold. }\end{array}$ & $\begin{array}{c}\text { Tempe- } \\
\text { rature of } \\
\text { the } \\
\text { water. }\end{array}$ & Time of & Remarks. \\
\hline I. & $\begin{array}{l}\text { Left } \\
\text { hand. }\end{array}$ & $\cdots$ & ... & ... & $96 \cdot 3^{\circ}$ & $\begin{array}{l}\text { Right } \\
\text { hand. }\end{array}$ & $46^{\circ}$ & 20 min. & Water felt ver \\
\hline II. & $\begin{array}{l}\text { Left } \\
\text { hand. }\end{array}$ & $\cdots$ & $95 \cdot 3^{\circ}$ & $953^{\circ}$ & $\cdots$ & $\begin{array}{l}\text { Right } \\
\text { hand. }\end{array}$ & $47^{\circ}$ & $10 \mathrm{~min}$. & Ditto. \\
\hline III. & $\begin{array}{l}\text { Left } \\
\text { hand. }\end{array}$ & $\cdots$ & $\cdots$ & $\cdots$ & $97 \cdot 6^{\circ}$ & $\begin{array}{l}\text { Right } \\
\text { hand. }\end{array}$ & $40^{\circ}$ & 30 min. & Ditto. \\
\hline IV. & $\begin{array}{l}\text { Right } \\
\text { hand. }\end{array}$ & $\cdots$ & $\cdots$ & $\cdots$ & $98^{\circ}$ & $\begin{array}{l}\text { Left } \\
\text { hand. }\end{array}$ & $36^{\circ}$ & $30 \mathrm{~min}$. & Pain considepal \\
\hline v. & $\begin{array}{l}\text { Right } \\
\text { hand. }\end{array}$ & $\cdots$ & $86^{\circ}$ & $89 \cdot 2^{\circ}$ & $94^{\circ}$ & $\begin{array}{l}\text { Left } \\
\text { hand. }\end{array}$ & $36^{\circ}$ & $25 \mathrm{~min}$. & 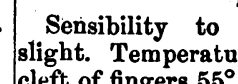 \\
\hline$\dot{\nabla} \mathbf{I}$. & $\begin{array}{l}\text { Left } \\
\text { hand. }\end{array}$ & $\cdots$ & $96 \cdot 2^{\circ}$ & $964^{\circ}$ & $96.4^{\circ}$ & $\begin{array}{l}\text { Right } \\
\text { hand. }\end{array}$ & $39^{\circ}$ & 20 min. & Hahd was with \\
\hline $\begin{array}{l}\text { vil. } \\
(a)\end{array}$ & $\begin{array}{l}\text { Right } \\
\text { hand. }\end{array}$ & ... & $\cdots$ & $\cdots$ & $96^{\circ}$ & $\begin{array}{l}\text { Left } \\
\text { hand. }\end{array}$ & $36^{\circ}$ & 15 min. & $\begin{array}{l}\text { as the pain was sev } \\
\text { Considerable pai } \\
\text { first, afterwards } \\
\text { bearable. Tempers } \\
\text { in cleft of fingers } 5\end{array}$ \\
\hline (b) & $\begin{array}{l}\text { Right } \\
\text { hand. }\end{array}$ & $\cdots$ & $\cdots$ & $\cdots$ & $93.4^{\circ}$ & $\begin{array}{l}\text { Left } \\
\text { hand. }\end{array}$ & $25^{\circ}$ & $.3 \mathrm{~min}$. & Pain moderate. \\
\hline (c) & $\begin{array}{l}\text { Right } \\
\text { hand. }\end{array}$ & $\cdots$ & $\cdots$ & $\cdots$ & $94: 6^{\circ}$ & $\begin{array}{l}\text { Left } \\
\text { hand. }\end{array}$ & $26^{\circ}$ & $6 \frac{1}{2} \mathrm{~min}$. & Ditto. \\
\hline VIII. & $\begin{array}{c}\text { Left } \\
\text { foot. } \\
\text { Mouth. }\end{array}$ & $\cdots$ & $\begin{array}{c}92 \cdot 8^{\circ} \\
\ldots\end{array}$ & $\begin{array}{l}95 \cdot 5^{\circ} \\
98^{\circ}\end{array}$ & $\begin{array}{r}95 \cdot 8 \\
98^{\circ}\end{array}$ & $\begin{array}{l}\text { Right } \\
\text { foot. }\end{array}$ & $56^{\circ}$ & $25 \mathrm{~min}$. & $\begin{array}{l}\text { Water felt cold } \\
\text { periment was me } \\
\text { March. }\end{array}$ \\
\hline$\underset{(a)}{\operatorname{IX}}$ & $\begin{array}{l}\text { Left } \\
\text { hand. } \\
\text { Mouth. }\end{array}$ & $\begin{array}{l}\cdots \\
\cdots\end{array}$ & $\begin{array}{c}95 \cdot 9^{\circ} \\
\ldots\end{array}$ & $\begin{array}{c}96 \cdot 6^{\circ} \\
\ldots\end{array}$ & $\begin{array}{l}96 \cdot 6^{\circ} \\
98 \cdot 2\end{array}$ & $\begin{array}{l}\text { Right } \\
\text { hand. }\end{array}$ & $49^{\circ}$ & $20 \mathrm{~min}$. & Water felt ver \\
\hline (b) & $\begin{array}{l}\text { Left } \\
\text { hand. }\end{array}$ & $\ldots$ & $\ldots$ & ... & $96 \cdot 4^{\circ}$ & ... & ... & $22 \mathrm{~min}$. & $\begin{array}{l}\text { Sat in draught } \\
\text { against the tap. }\end{array}$ \\
\hline $\mathbf{x}$. & $\begin{array}{c}\text { Left } \\
\text { hand. } \\
\text { Mouth. }\end{array}$ & $\cdots$ & $98 \cdot 8^{\circ}$ & $97 \cdot 2^{\circ}$ & $98 \cdot 2^{\circ}$ & $\begin{array}{l}\text { Right } \\
\text { hand. }\end{array}$ & $36^{\circ}$ & $19 \mathrm{~min}$. & $\begin{array}{l}\text { Intense pain for } \\
\text { seconds, more bear } \\
\text { afterwards. }\end{array}$ \\
\hline XI. & $\begin{array}{l}\text { Right } \\
\text { hand. } \\
\text { Mouth }\end{array}$ & $\cdots$ & $\mid \begin{array}{c}\cdots \\
98 \cdot 8^{\circ}\end{array}$ & $92 \cdot 5^{\circ}$ & $\left|\begin{array}{c}98 \cdot 2^{\circ} \\
\ldots\end{array}\right|$ & $\begin{array}{l}\text { Eeft } \\
\text { hand. }\end{array}$ & $36^{\circ}$ & $14 \mathrm{~min}$. & $\begin{array}{l}\text { Only moderately } \\
\text { ful at first. }\end{array}$ \\
\hline
\end{tabular}




\begin{tabular}{|c|c|c|c|c|c|c|}
\hline \multicolumn{7}{|c|}{ OBSERVATION OF TEMPERATURE SUBSEQUENT TO APPLICATION OF COLD } \\
\hline $\begin{array}{l}\text { Temp. } \\
\text { T-2 min. } \\
\text { after ap- } \\
\text { aflication } \\
\text { of cold. }\end{array}$ & $\left|\begin{array}{c}\text { Temp. } \\
\text { Te-6 min. } \\
\text { after ap- } \\
\text { plication } \\
\text { of cold. }\end{array}\right|$ & $\begin{array}{l}\text { Temp. } \\
\text { - - } 10 \text { min. } \\
\text { after ap- } \\
\text { plication } \\
\text { of cold. }\end{array}$ & $\begin{array}{l}\text { Temp. } 12 \\
\text {-15 min. } \\
\text { after ap- } \\
\text { plication } \\
\text { of cold. }\end{array}$ & $\begin{array}{l}\text { Temp. } 20 \\
-30 \text { min. } \\
\text { after ap- } \\
\text { plication } \\
\text { of cold. }\end{array}$ & \begin{tabular}{|c|} 
Greatest \\
altera- \\
tion of \\
tempera- \\
ture.
\end{tabular} & Remarks. \\
\hline$\cdots$ & $96 \cdot 2^{\circ}$ & $96 \cdot 3^{\circ}$ & $96 \cdot 2^{\circ}$ & $96 \cdot 2^{\circ}$ & $-0 \cdot 1^{\circ}$ & $\begin{array}{l}\text { Hand grasping the thermometer and } \\
\text { no bandage used }\end{array}$ \\
\hline ... & $95 \cdot 4^{\circ}$ & $96 \cdot 3^{\circ}$ & $\cdots$ & $\cdots$ & $+\cdot 0^{\circ}$ & Ditto \\
\hline$\cdots$ & $\cdots$ & $\cdots$ & $97 \cdot 7^{\circ}$ & $97 \cdot 7^{\circ}$ & $+1^{\circ}$ & A bandage was placed round the fist \\
\hline$\rightarrow \cdots$ & $\cdots$ & $98 \cdot 2^{\circ}$ & $\cdots$ & $98 \cdot 1^{\circ}$ & $+0.2^{3}$ & Ditto \\
\hline$\cdots$ & $94: 4^{\circ}$ & $95 \cdot 8^{\circ}$ & $96 \cdot 2^{\circ}$ & $96^{\circ}$ & $+2 \cdot 2^{\circ}$ & Ditto \\
\hline ... & $96 \cdot 2^{\circ}$ & $96.5^{\circ}$ & $96 \cdot 8^{\circ}$ & $96 \cdot 8^{\circ}$ & $+0.4^{\circ}$ & Fist was supported by an elastic band \\
\hline $95 \cdot 9^{\circ}$ & $96^{\circ}$ & ... & $96.4^{\circ}$ & $96^{\circ}$ & $+0.4^{\circ}$ & $\begin{array}{l}\text { This observation was made on the } \\
\text { same individual as } V \text {, but in this case } \\
\text { she was somewhat exhausted. Direatly } \\
\text { after removing her hand from the water } \\
\text { she felt chilly, and the temperature fell } \\
\text { in twenty-five minutes to } 92 \cdot 8^{\circ} \text {, rising } \\
\text { shortly after to } 93 \cdot 4^{\circ} \text {. Another observa- } \\
\text { tion was made (b). }\end{array}$ \\
\hline$\cdots$ & $93 \cdot 4^{\circ}$ & $\cdots$ & $\cdots$ & ... & $0^{\circ}$ & $\begin{array}{l}\text { The temperature continued to rise } \\
\text { when the hand was withdrawn from the } \\
\text { freezing mixture. Obs. (c) was then }\end{array}$ \\
\hline $94: 8^{\circ}$ & $94: 8^{\circ}$ & $94: 6^{\circ}$ & $\ldots$ & $\ldots$ & $+0.2^{\circ}$ & $\begin{array}{l}\text { Chilliness again came on, with a sen- } \\
\text { sation of cold down the back, the tem- } \\
\text { perature falling to } 93^{\circ} \text {. A cup of hot } \\
\text { tea was given, soon after which the } \\
\text { chilliness passed off and the temperature } \\
\text { rose. }\end{array}$ \\
\hline $95 \cdot 7^{\circ}$ & $95 \cdot 7^{\circ}$ & $96^{\circ}$ & $96 \cdot 1^{\circ}$ & $96 \cdot 2^{\circ}$ & $+0 \cdot 4^{\circ}$ & $\begin{array}{l}\text { Bulb of thermometer placed under } \\
\text { toes and foot lightly covered. }\end{array}$ \\
\hline ... & $\cdots$ & $\cdots$ & $\cdots$ & $98^{\circ}$ & $0^{\circ}$ & \\
\hline$\ldots$ & $96 \cdot 2^{\circ}$ & $\ldots$ & $\ldots$ & $95.6^{\circ}$ & $-1 \cdot 0^{\circ}$ & The subect of this experiment moved \\
\hline$\cdots$ & $\cdots$ & $\cdots$ & $\cdots$ & $98 \cdot 2^{\circ}$ & ... & $\begin{array}{l}\text { to a tap of water which stood in a slight } \\
\text { draught. No chilliness. See }(b) \text {. }\end{array}$ \\
\hline$\cdots$ & $\ldots$ & $\cdots$ & $96.2^{\circ}$ & $95 \cdot 2^{\circ}$ & $-1 \cdot 2^{\circ}$ & $\begin{array}{l}\text { In this case the right hand was not } \\
\text { put into water, but an equal fall of tem- } \\
\text { perature occurred in a space of time }\end{array}$ \\
\hline $98 \cdot 2^{\circ}$ & $98 \cdot 3^{\circ}$ & $98.4^{\circ}$ & $98 \cdot 4^{\circ}$ & $98 \cdot 4^{\circ}$ & $+0.2^{\circ}$ & $\begin{array}{l}\text { This experiment and Experiment XI } \\
\text { were made on two healthy adult males } \\
\text { who had recently dined. The results } \\
\text { in XI were almost precisely similar to } \\
\text { those in X }\end{array}$ \\
\hline $98 \cdot 1^{\circ}$ & $98 \cdot 0^{\circ}$ & $98 \cdot 2^{\circ}$ & $98 \cdot 3$ & $\cdots$ & $+0 \cdot 1^{\circ}$ & ... \\
\hline$\cdots$ & ... & $\cdots$ & $99 \cdot 0$ & $\cdots$ & $\left|+0.2^{\circ}\right|$ & \\
\hline
\end{tabular}




\begin{tabular}{|c|c|c|c|c|c|c|c|c|c|c|c|c|c|c|c|c|c|c|c|c|c|c|c|c|c|c|c|c|}
\hline \multicolumn{4}{|c|}{ 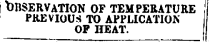 } & \multicolumn{4}{|c|}{ APPLICATION OP HEAT. } & \multicolumn{8}{|c|}{$\begin{array}{l}\text { ODSERVADN OP TEMPERATURE SUBSEQUENT TO APPLICATION } \\
\text { OF HEAT. }\end{array}$} & \multicolumn{4}{|c|}{ APPLICATION OF COLD. } & \multicolumn{9}{|c|}{ OBSERVATION OF TEMPERATURE SUBSEQUENT TO APPLICATION OF COLD. } \\
\hline Experinent & $\mid \begin{array}{c}\text { Part of hody } \\
\text { in which } \\
\text { tempera- } \\
\text { tume was } \\
\text { observed. }\end{array}$ & $\begin{array}{l}\text { al } \\
\text { Tempe } \\
\text { reture at } \\
\text { end of } \\
\text { manutes. } \\
\end{array}$ & 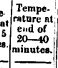 & $\begin{array}{c}\text { Name of } \\
\text { extremity } \\
\text { inmersed } \\
\text { in hoc } \\
\text { mater. } \\
\end{array}$ & $\begin{array}{l}\text { Tempe } \\
\text { rature of } \\
\text { anter. } \\
\text { ant }\end{array}$ & of Timen of & Remarks. & 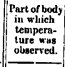 & 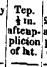 & 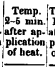 & 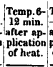 & 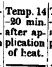 & 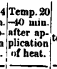 & 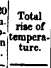 & Remarks. & 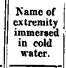 & $\begin{array}{l}\text { Tempe } \\
\text { rature of } \\
\text { water. }\end{array}$ & $\begin{array}{l}\text { Time of } \\
\text { immersion. }\end{array}$ & Remarka. & 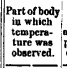 & 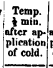 & 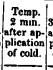 & 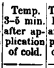 & 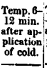 & 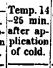 & 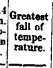 & \begin{tabular}{|c|c|}
$\begin{array}{c}\text { Fell } \\
\text { below } \\
\text { nerman } \\
\text { tempora. } \\
\text { ture. }\end{array}$ \\
\end{tabular} & Remarks. \\
\hline XII. & $\begin{array}{l}\text { Left } \\
\text { hand. }\end{array}$ & $926^{\circ}$ & $95.8^{\circ}$ & $\begin{array}{l}\text { Right } \\
\text { hand. }\end{array}$ & $1120^{\circ}$ to & $35 \mathrm{~min}$. & 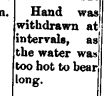 & \begin{tabular}{|l|} 
Left \\
band. \\
\end{tabular} & 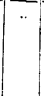 & $96^{\circ}$ & $96 \cdot 6^{\circ}$ & 97 & $\overline{97 \cdot 2^{\circ}}$ & $\overline{1.4^{\circ}}$ & $\mid \begin{array}{c}\text { No fall of } \\
\text { temperature } \\
\text { occurred on } \\
\text { the first appli- } \\
\text { cation of heat. }\end{array}$ & & & & & & & & & & & & & \\
\hline XIII. & $\begin{array}{l}\text { Right } \\
\text { hand. } \\
\text { Mouth. }\end{array}$ & $\cdots$ & $\mid \begin{array}{l}97 \cdot 3^{\circ} \\
98 \cdot 4^{\circ}\end{array}$ & $\begin{array}{l}\text { Left } \\
\text { hand. }\end{array}$ & $\mid \begin{array}{c}110^{\circ} \text { to } \\
120^{\circ}\end{array}$ & 35 miv. & a. $\begin{array}{c}\text { Hand with- } \\
\text { Irawn for six } \\
\text { minutes at } \\
\text { middleoftime. }\end{array} \mid$ & $\begin{array}{c}\text { Right } \\
\text { band. } \\
\text { Mouth. }\end{array}$ & .. & $97 \cdot 2^{\circ}$ & $\begin{array}{c}97 \cdot 8^{\circ} \\
\ldots\end{array}$ & $98 \cdot 6^{\circ}$ & $\mid \begin{array}{l}99 \cdot 4^{\circ} \\
99 \cdot 4^{\circ}\end{array}$ & \begin{tabular}{|c|}
$2 \cdot 1^{\circ}$ \\
$110^{\circ}$
\end{tabular} & $\mid$\begin{tabular}{c|} 
Tempera- \\
ture fello-1 ion \\
first applica- \\
tion of heat.
\end{tabular} & $\begin{array}{l}\text { Left } \\
\text { hand. }\end{array}$ & $\begin{array}{c}45^{\circ} \text { to } \\
54^{\circ}\end{array}$ & $25 \mathrm{~min}$. & $\cdots$ & \begin{tabular}{|c} 
Right \\
hand. \\
Mouth.
\end{tabular} & $99 \cdot 4^{\circ}$ & $99 \cdot 3^{\circ}$ & $98 \cdot 8^{\circ}$ & $98 \cdot 2^{c}$ & $\begin{array}{l}96 \cdot 4^{\circ} \\
98 \cdot 4^{\circ}\end{array}$ & $\begin{array}{c}3.0^{\circ} \\
1.0^{\circ}\end{array}$ & $0.9^{\circ}$ & $\begin{array}{l}\text { A feeling of chilliniess came } \\
\text { on soon after immersion of the } \\
\text { hand in cold water, and in- } \\
\text { creased in intensity. }\end{array}$ \\
\hline XIV. & $\begin{array}{c}\text { Left } \\
\text { land. } \\
\text { Month. }\end{array}$ & $\cdots$ & $\begin{array}{l}91 \cdot 2^{\circ} \\
98 \cdot 4^{\circ}\end{array}$ & $\begin{array}{l}\text { Right } \\
\text { hand. }\end{array}$ & $\left|\begin{array}{c}115^{\circ} \text { to } \\
120^{\circ}\end{array}\right|$ & $31 \mathrm{~min}$. & a. $\begin{array}{c}\text { Hand con- } \\
\text { tinuously im. } \\
\text { mersed. Temp. } \\
\text { of room } 66^{\circ} .\end{array}$ & $\begin{array}{c}\text { Left } \\
\text { hand. } \\
\text { Mouth. }\end{array}$ & $94{ }^{\circ}$ & $94 \cdot 6^{\circ}$ & $\mid \begin{array}{c}95 \cdot 8^{\circ} \\
\cdots\end{array}$ & $\begin{array}{c}96 \cdot 6^{\circ} \\
\cdots\end{array}$ & $\left|\begin{array}{l}97 \cdot 6^{\circ} \\
98 \cdot 7^{\circ}\end{array}\right|$ & $\mid \begin{array}{l}3.4^{\circ} \\
0.3^{\circ}\end{array}$ & \begin{tabular}{|l|} 
No primary \\
fall of tempe- \\
rature occur- \\
red. Temp. of \\
room $66^{\circ} 0^{\circ}$
\end{tabular} & $\begin{array}{c}\text { Right } \\
\text { band. } \\
\end{array}$ & $53^{\circ}$ & $23 \mathrm{~min}$. & $\cdots$ & $\begin{array}{c}\text { Left } \\
\text { hand. } \\
\text { Mouth. }\end{array}$ & $\ldots$ & $\begin{array}{c}97 \cdot 6^{\circ} \\
\cdots\end{array}$ & $97 \cdot 4^{\circ}$ & $97 \cdot 2^{\circ}$ & $\mid \begin{array}{l}97 \cdot 0^{\circ} \\
98 \cdot 0^{\circ}\end{array}$ & $\left|\begin{array}{c}0.6^{\circ} \\
0.7^{\circ}\end{array}\right|$ & $\mathbf{0} \mathbf{4}^{\circ}$ & $\begin{array}{l}\text { Chilliness came on after hand } \\
\text { was dried, and in } 30 \text { minutes } \\
\text { temperature in left hand was } \\
\text { 95.4, and in mouth } 97 \cdot 6 \text {; after } \\
\text { some hot tea the temperature } \\
\text { rose. }\end{array}$ \\
\hline Xv. & $\begin{array}{c}\text { Left } \\
\text { foot. } \\
\text { Mouth. }\end{array}$ & $92 \cdot 5^{\circ}$ & $95 \cdot 8^{\circ}$ & $\begin{array}{c}\text { Right } \\
\text { foot. }\end{array}$ & $\left|\begin{array}{r}108^{\circ} \text { to } \\
114^{\circ}\end{array}\right|$ & $21 \mathrm{~min}$. & - $\begin{array}{c}\text { Foot con- } \\
\text { tinuously } \\
\text { mersed. }\end{array} \mid$ & $\begin{array}{c}\text { Left } \\
\text { foot. } \\
\text { Mouth. }\end{array}$ & $\ldots$ & $\cdots$ & $96.4^{\circ}$ & $97.8^{\circ}$ & $\ldots$ & $\begin{array}{c}1.0^{\circ} \\
0^{\circ}\end{array}$ & $\left|\begin{array}{c}\text { Foot } \\
\text { ported by } \\
\text { elastic band. }\end{array}\right|$ & $\begin{array}{l}\text { Right } \\
\text { foot. }\end{array}$ & $55^{\circ}$ & $32 \mathrm{~min}$. & Felt very: & $\begin{array}{c}\text { Left } \\
\text { foot. } \\
\text { Mouth. }\end{array}$ & $\begin{array}{c}96-9^{\circ} \\
\ldots\end{array}$ & $\cdots$ & $96.9^{\circ}$ & $96 \cdot 9^{\circ}$ & $\begin{array}{l}96 \cdot 9^{\circ} \\
97 \cdot 8^{\circ}\end{array}$ & \begin{tabular}{l|l}
$\circ$ & $\ldots$
\end{tabular} & $\ldots$ & $\begin{array}{l}\text { The temperature of the left } \\
\text { foot rose continuously, not- } \\
\text { withstanding the moderate } \\
\text { cold applied to the right foot. }\end{array}$ \\
\hline XVI. & $\begin{array}{c}\begin{array}{c}\text { Left } \\
\text { hand. } \\
\text { Mouth. }\end{array} \\
\end{array}$ & $97 \cdot 3^{\circ}$ & $97 \cdot 6^{\circ}$ & $\begin{array}{l}\text { Right } \\
\text { hand. }\end{array}$ & $\left|\begin{array}{c}116^{\circ} \text { to } \\
120^{\circ}\end{array}\right|$ & $14 \mathrm{~min}$. & a. $\begin{array}{c}\text { Hand con- } \\
\text { tinuously im- } \\
\text { mersed. Tem. } \\
\text { perature of } \\
\text { room } 69.0^{\circ} .\end{array} \mid$ & $\begin{array}{c}\text { Left } \\
\text { hand. } \\
\text { Mouth. }\end{array}$ & $\ldots$ & $\begin{array}{c}\mathbf{9 7 \cdot 4 ^ { \circ }} \\
\\
\cdots\end{array}$ & 98 & $\mid \begin{array}{l}98 \cdot 5^{\circ} \\
99 \cdot 2^{\circ}\end{array}$ & $\ldots$ & $0.9^{\circ}$ & \begin{tabular}{l|} 
A primary \\
fall of $0 \cdot 2^{\circ}$ oc. \\
curred. Tom. \\
perature bc. \\
gan to fall as \\
soon us hand \\
sas removed \\
was hom hot water \\
being at that \\
time $98^{\circ} \cdot 2^{\circ}$.
\end{tabular} & \begin{tabular}{|c|} 
Right \\
hand.
\end{tabular} & $50^{\circ}$ to & $27 \mathrm{~min}$. & 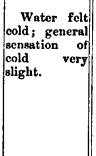 & $\begin{array}{c}\text { Left } \\
\text { hand. } \\
\text { Mouth. }\end{array}$ & $\begin{array}{l}\cdots \\
\cdots\end{array}$ & $\begin{array}{l}98^{\circ} \\
\ldots\end{array}$ & $\begin{array}{l}97^{\circ} \\
\cdots\end{array}$ & $95^{\circ}$ & $\begin{array}{c}92 \cdot 5^{\circ} \\
98 \cdot 6^{\circ}\end{array}$ & $\begin{array}{l}6.0^{\circ} \\
0.6^{\circ}\end{array}$ & $5 \cdot 1^{\circ}$ & $\begin{array}{l}\text { The temperature fell another } \\
1^{\circ} \text { after the band was dried, } \\
\text { but rose rapidly on reimumer- } \\
\text { sion in hot water; left hand } \\
\text { and body were sweating whilst } \\
\text { right was in hot water, but } \\
\text { became dry when it was placed } \\
\text { in cold. }\end{array}$ \\
\hline XVII. & $\begin{array}{l}\text { Right } \\
\text { hand. } \\
\text { Month. }\end{array}$ & $\begin{array}{l}94^{\circ} \\
98^{\circ}\end{array}$ & 96-2 $2^{\circ}$ & $\begin{array}{l}\text { Left } \\
\text { hand. }\end{array}$ & $108^{\circ} \mathrm{to}$ & $19 \mathrm{~min}$. & . $\begin{array}{c}\text { Hand con. } \\
\text { tinuoualy in. } \\
\text { mersed. }\end{array}$ & $\begin{array}{c}\text { Right } \\
\text { hand. } \\
\text { Moath. }\end{array}$ & $\begin{array}{l}\cdots \\
.\end{array}$ & $\begin{array}{c}96 \cdot 2^{\circ} \\
\ldots\end{array}$ & \begin{tabular}{l|l}
$97^{\circ}$ \\
$\cdots$
\end{tabular} & $\begin{array}{c}98^{\circ} \\
98 \cdot 8^{\circ}\end{array}$ & $\cdots$ & $\begin{array}{l}1.8^{\circ} \\
0.8^{\circ}\end{array}$ & $\mid \begin{array}{l}\text { Hand grasp- } \\
\text { ing thermo- } \\
\text { meter. }\end{array}$ & $\cdots$ & $\cdots$ & $\cdots$ & $\cdots$ & $\cdots$ & $\cdots$ & $\cdots$ & $\cdots$ & $\cdots$ & $\cdots$ & $\cdots$ & $\cdots$ & $\begin{array}{l}\text { Subject of experiment was a } \\
\text { feeble old man. }\end{array}$ \\
\hline xviII. & $\begin{array}{c}\text { Right } \\
\text { band. } \\
\text { Mouth. }\end{array}$ & $\left|\begin{array}{c}92 \cdot 8^{\circ} \\
98 \cdot 8^{\circ}\end{array}\right|$ & $92.0^{\circ}$ & Left foot. & $\mid \begin{aligned} 111^{\circ} \\
118^{\circ}\end{aligned}$ & $24 \mathrm{~min}$ & 2. $\begin{array}{c}\text { Foot con- } \\
\text { tinuonsly im- } \\
\text { mersed. }\end{array}$ & \begin{tabular}{|l|} 
Right \\
hand. \\
Mouth.
\end{tabular} & $88 \div 1^{\circ}$ & $\ldots$ & $\begin{array}{c}87 \cdot 6^{\circ} \\
\cdots\end{array}$ & $\begin{array}{l}88 \cdot 6^{\circ} \\
99 \cdot 2^{\circ}\end{array}$ & $\begin{array}{c}91 \cdot 4^{\circ} \\
\cdots\end{array}$ & $0 \cdot 4^{\circ}$ & $\begin{array}{l}\text { A primary } \\
\text { fall of nearly } \\
4^{\circ} \text {. No sensan. } \\
\text { tion of chilli. } \\
\text { pess oattended } \\
\text { it. }\end{array}$ & $\begin{array}{l}\text { Left } \\
\text { foot. }\end{array}$ & $\mid \begin{array}{c}50^{\circ} \text { to } \\
55^{\circ}\end{array}$ & $20 \mathrm{~min}$. & $\begin{array}{l}\text { Slight feel- } \\
\text { ing of chilli- } \\
\text { ness. }\end{array}$ & $\begin{array}{l}\text { Right } \\
\text { hand. } \\
\text { Mouth. }\end{array}$ & $\begin{array}{c}91 \cdot 4^{\circ} \\
\ldots\end{array}$ & $\begin{array}{c}91 \cdot 2^{\circ} \\
\ldots\end{array}$ & $\begin{array}{c}90 \cdot 6^{\circ} \\
\ldots\end{array}$ & $89 \cdot 4^{\circ}$ & $\left|\begin{array}{l}87 \cdot 6^{\circ} \\
98 \cdot \theta^{\circ}\end{array}\right|$ & $\begin{array}{c}3.8^{\circ} \\
0.3^{\circ}\end{array}$ & $44^{\circ}$ & $\begin{array}{l}\text { The subject of this experi- } \\
\text { ment had recently recovered } \\
\text { from a long illness. }\end{array}$ \\
\hline XIX. & $\begin{array}{c}\text { Left } \\
\text { hand. } \\
\text { Left foot. } \\
\text { Moutb. }\end{array}$ & $\mid \begin{array}{c}96 \cdot 1^{\circ} \\
95^{\circ} \\
98 \cdot 4^{\circ}\end{array}$ & $\left|\begin{array}{l}97 \cdot 0^{\circ} \\
96 \cdot 1^{\circ}\end{array}\right|$ & $\begin{array}{l}\text { Right } \\
\text { hand. }\end{array}$ & $118^{\circ}$ to & $21 \mathrm{~min}$. & $\mid \begin{array}{c}\text { Hand con. } \\
\text { tinuously im. } \\
\text { mersed. }\end{array}$ & $\begin{array}{c}\text { Left } \\
\text { haud. } \\
\text { Left foot. } \\
\text { Mouth. }\end{array}$ & $\begin{array}{l}97 \\
\ldots \\
\ldots\end{array}$ & $\begin{array}{c}97 \\
96 \cdot 2^{\circ} \\
\ldots \\
\end{array}$ & \begin{tabular}{c|c}
$97 \cdot 5^{\circ}$ & 8 \\
$97 \cdot 2^{\circ}$ & 8 \\
$\ldots$ & 8
\end{tabular} & $\begin{array}{l}98 \cdot 0^{\circ} \\
98 \cdot 0^{\circ} \\
98 \cdot 9^{\circ}\end{array}$ & $\ldots$ & $\begin{array}{l}1.0^{\circ} \\
1.8^{\circ} \\
0.5^{\circ}\end{array}$ & $\ldots$ & $\begin{array}{l}\text { Right } \\
\text { hand. }\end{array}$ & $55^{\circ}$ & $21 \mathrm{~min}$. & $\cdots$ & $\begin{array}{c}\begin{array}{c}\text { Left } \\
\text { hand. } \\
\text { Left foot. } \\
\text { Moath. }\end{array} \\
\end{array}$ & $\begin{array}{l}98^{\circ} \\
98^{\circ} \\
\ldots\end{array}$ & $\begin{array}{l}\cdots \\
\cdots \\
\cdots\end{array}$ & $\begin{array}{l}\cdots \\
\cdots \\
\cdots\end{array}$ & $\begin{array}{c}97 \cdot 6^{\circ} \\
97 \cdot 2^{\circ} \\
\ldots \\
\end{array}$ & $\left|\begin{array}{l}97 \cdot 2^{\circ} \\
97 \cdot 0^{\circ} \\
97 \cdot 9^{\circ}\end{array}\right|$ & $\begin{array}{l}0.8^{\circ} \\
1.0^{\circ} \\
1.0^{\circ}\end{array}$ & $0.5^{\circ}$ & $\begin{array}{l}\text { No fall below the normal } \\
\text { occurred in this case from the } \\
\text { application of cold. }\end{array}$ \\
\hline $\mathbf{x x}$. & $\begin{array}{c}\text { Left } \\
\text { hand. } \\
\text { Mouth. }\end{array}$ & $\left|\begin{array}{c}\cdots \\
98 \cdot 4^{\circ}\end{array}\right|$ & $\left|96.5^{\circ}\right|$ & $\begin{array}{l}\text { Right } \\
\text { hand. }\end{array}$ & $\mid \begin{aligned} & 118^{\circ} \text { to } \\
& 122^{\circ}\end{aligned}$ & $83 \mathrm{~min}$ & ... & $\begin{array}{c}\text { Left } \\
\text { hand. } \\
\text { Mouth. }\end{array}$ & $\cdots$ & $\begin{array}{c}96 \cdot 4^{\circ} \\
\ldots\end{array}$ & $\begin{array}{c}96 \cdot 8^{\circ} \\
\cdots\end{array}$ & $\begin{array}{c}97 \cdot 9^{\circ} \\
\ldots\end{array}$ & $\left|\begin{array}{l}98 \cdot 2^{\circ} \\
99 \cdot 0^{\circ}\end{array}\right|$ & $\begin{array}{l}1.7^{\circ} \\
0.6^{\circ}\end{array}$ & $\ldots$ & $\begin{array}{l}\text { Right } \\
\text { hand. }\end{array}$ & \begin{tabular}{l|l}
$88^{\circ}$ & 1
\end{tabular} & 14 min. & \begin{tabular}{|} 
Much pain; \\
hand & with. \\
drawn & now \\
and then.
\end{tabular} & $\begin{array}{c}\text { Left } \\
\text { hand. } \\
\text { Month. }\end{array}$ & $\cdots$ & $\ldots$ & $\begin{array}{c}97 \cdot 8^{\circ} \\
\ldots\end{array}$ & $\begin{array}{c}97 \cdot 2^{\circ} \\
\ldots\end{array}$ & $\left|\begin{array}{l}96 \cdot 2^{\circ} \\
97 \cdot 6^{\circ}\end{array}\right|$ & $\begin{array}{c}2 \cdot 0^{\circ} \\
1 \cdot 4^{\circ}\end{array}$ & $0.8^{\circ}$ & 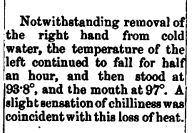 \\
\hline XXI. & $\begin{array}{c}\text { Left } \\
\text { band. } \\
\text { Mouth. }\end{array}$ & $\ldots$ & $\left|\begin{array}{l}97 \cdot 2^{\circ} \\
98 \cdot 4^{\circ}\end{array}\right|$ & $\begin{array}{l}\text { Right } \\
\text { foot. }\end{array}$ & $118^{\circ}$ to & of $43 \mathrm{~min}$ & $\begin{array}{l}\text { Foot with. } \\
\text { drawn at in- } \\
\text { tervals, as the } \\
\text { water was very } \\
\text { hot. }\end{array}$ & \begin{tabular}{|c|} 
Left \\
hand. \\
Mouth.
\end{tabular} & $\cdots$ & $\cdots$ & $\begin{array}{c}97 \cdot 4^{\circ} \\
\ldots\end{array}$ & $\begin{array}{c}97 \cdot 8^{\circ} \\
\ldots\end{array}$ & $\mid \begin{array}{l}98 \cdot 0^{\circ} \\
98 \cdot 8^{\circ}\end{array}$ & $\begin{array}{l}0.8^{\circ} \\
0.4^{\circ}\end{array}$ & $\begin{array}{l}\text { Hand grasp- } \\
\text { ing thermo- } \\
\text { meter. Gene- } \\
\text { ral feeling of } \\
\text { beat. }\end{array}$ & $\begin{array}{c}\text { Right } \\
\text { hand or } \\
\text { right } \\
\text { foot. }\end{array}$ & $36^{\circ}$ & $47 \mathrm{~min}$. & $\begin{array}{c}\text { Sensation of } \\
\text { pain replaced } \\
\text { that of cold. }\end{array} \mid$ & $\begin{array}{c}\text { Left } \\
\text { hand. } \\
\text { Month. }\end{array}$ & $\cdots$ & $\begin{array}{l}\cdots \\
\cdots\end{array}$ & $\cdots$ & $\begin{array}{c}97 \cdot 8^{\circ} \\
\ldots\end{array}$ & $\begin{array}{l}97 \cdot 2^{\circ} \\
98 \cdot 0^{\circ}\end{array}$ & $\left(\begin{array}{c}0.8^{\circ} \\
0.8^{\circ}\end{array}\right.$ & \begin{tabular}{c|}
$0^{\circ}$ \\
$0.4^{\circ}$
\end{tabular} & \\
\hline XXII. & $\begin{array}{c}\begin{array}{c}\text { Left } \\
\text { hand. } \\
\text { Mouth. }\end{array} \\
\text {. }\end{array}$ & $\cdots$ & $\begin{array}{l}98.3^{\circ} \\
99.0^{\circ}\end{array}$ & $\begin{array}{l}\text { Right } \\
\text { foot. }\end{array}$ & $116^{\circ}$ to & $28 \mathrm{~min}$ & $\begin{array}{l}\text { Foot con. } \\
\text { tinnously im- } \\
\text { mersed. }\end{array}$ & $\begin{array}{c}\text { Left } \\
\text { band. } \\
\text { Mouth. }\end{array}$ & $\cdots$ & \begin{tabular}{c|}
$98 \cdot 3^{\circ}$ \\
$\ldots$
\end{tabular} & $\begin{array}{c}98 \cdot 6^{\circ} \\
\ldots\end{array}$ & $\begin{array}{c}99 \cdot 0^{\circ} \\
\ldots\end{array}$ & $\begin{array}{l}99 \cdot 1^{\circ} \\
99 \cdot 6^{\circ}\end{array}$ & $\begin{array}{c}0.8^{\circ} \\
0.6^{\circ}\end{array}$ & $\cdots$ & $\begin{array}{l}\text { Right } \\
\text { foot. }\end{array}$ & $38^{\circ}$ & $40 \mathrm{~min}$. & $\ldots$ & $\begin{array}{c}\begin{array}{c}\text { Left } \\
\text { hand. } \\
\text { Mouth. }\end{array} \\
\text {. }\end{array}$ & \begin{tabular}{l|l}
$\ldots$ & 9 \\
$\ldots$ &
\end{tabular} & $99 \cdot 1^{\circ}$ & $99 \cdot 0^{\circ}$ & $\begin{array}{c}98 \cdot 7^{\circ} \\
\ldots\end{array}$ & $\left|\begin{array}{l}98 \cdot 3^{\circ} \\
98 \cdot 7^{\circ}\end{array}\right|$ & $\begin{array}{l}0.8^{\circ} \\
0.8^{\circ}\end{array}$ & $\begin{array}{c}0^{\circ} \\
0.3^{\circ}\end{array}$ & $\begin{array}{l}\text { This experiment and expe- } \\
\text { riment XXIII were made at } \\
\text { the samo time, under preciealy } \\
\text { similar conditions. }\end{array}$ \\
\hline XXIII. & $\begin{array}{c}\text { Right } \\
\text { hand. } \\
\text { Mouth. }\end{array}$ & $\begin{array}{l}\cdots \\
\cdots\end{array}$ & $\begin{array}{l}98 \cdot 5^{\circ} \\
99 \cdot 0^{\circ}\end{array}$ & $\begin{array}{l}\text { Left } \\
\text { foot. }\end{array}$ & $\left|\begin{array}{c}116^{\circ} \text { to } \\
120^{\circ}\end{array}\right|$ & $03 \mathrm{~min}$. & $\begin{array}{l}\text { Foot con-| } \\
\text { tinuously im- } \\
\text { mersed. Much } \\
\text { pain; slight } \\
\text { sweating. }\end{array}$ & $\begin{array}{c}\text { Right } \\
\text { band. } \\
\text { Mouth. }\end{array}$ & $\cdots$ & $\begin{array}{c}98 \cdot 8^{\circ} \\
\ldots\end{array}$ & $\begin{array}{c}98 \cdot 8^{\circ} \\
\ldots\end{array}$ & \begin{tabular}{c|}
$\mathbf{9 9 \cdot} \cdot \mathbf{2}^{\circ}$ \\
$\ldots$
\end{tabular} & $\mid \begin{array}{l}99 \cdot 4^{\circ} \\
99 \cdot 6^{\circ}\end{array}$ & $\begin{array}{l}0.9^{\circ} \\
0.6^{\circ}\end{array}$ & $\left|\begin{array}{c}\text { Hand merely } \\
\text { grasping } \\
\text { thermometer. }\end{array}\right|$ & $\begin{array}{l}\text { Left } \\
\text { foot. }\end{array}$ & $38^{\circ}$ & $34 \mathrm{~min}$. & $\cdots$ & $\begin{array}{c}\text { Right } \\
\text { hand. } \\
\text { Mouth. }\end{array}$ & $\cdots$ & \begin{tabular}{c|c}
$99 \cdot 2^{\circ}$ \\
$\ldots$
\end{tabular} & $\begin{array}{c}98 \cdot 8^{\circ} \\
\ldots\end{array}$ & $\begin{array}{c}98 \cdot 6^{\circ} \\
\ldots\end{array}$ & $\mid \begin{array}{l}98 \cdot 2^{\circ} \\
98 \cdot 8^{\circ}\end{array}$ & $\begin{array}{c}1.2^{\circ} \\
0.8^{\circ}\end{array}$ & $\begin{array}{l}0.8^{\circ} \\
0.8^{\circ}\end{array}$ & $\begin{array}{l}\text { The anbject of this experi- } \\
\text { ment was in robust healtb. }\end{array}$ \\
\hline
\end{tabular}

